\title{
Diffusion of Biodiesel in Rubber and the Resulting Mechanical Response under Cyclic Loading
}

\author{
Chai Ai Bao ${ }^{1, a}$, Andri Andriyana ${ }^{1, b}$, Erwan Verron ${ }^{2, \mathrm{c}}$ and Mohd. Rafie Johan ${ }^{1, \mathrm{~d}}$ \\ ${ }^{1}$ Deparment of Mechanical Engineering, University of Malaya, 50603 Kuala Lumpur, Malaysia. \\ ²UUNAM Université, École Centrale de Nantes, GeM, UMR CNRS 6183, BP 92101, 44321 Nantes, \\ France \\ achaiaibao@siswa.um.edu.my, ${ }^{\mathrm{b}}$ andri.andriyana@um.edu.my, cerwan.verron@ec-nantes.fr, \\ dmrafie@um.edu.my
}

Keywords: Diffusion, biodiesel, rubber, mechanical response, cyclic loading.

\begin{abstract}
The benefits of biodiesel to the economy and the environment have motivated increasing interest among researchers to study the feasible utilization of this fuel. The majority of the research focuses on the production of biodiesel and the simple compatibility tests of the engine components with biodiesel. The effect of palm biodiesel diffusion on the mechanical responses of swollen rubber is rarely explored. This paper describes a simple experimental procedure to conduct a stress free diffusion test. Moreover, the mechanical responses are evaluated.
\end{abstract}

\section{Introduction}

Biodiesel has been introduced as a potential supplement to commercial diesel due to the fact that it is environmental friendly and economically beneficial. Palm biodiesel, which is derived from plant and animal fat, provides better engine efficiency and produces a cleaner environment. The compatibility issue of the engine components, particularly the rubber components has led to increasing interest in the research work of this kind [1-3]. However, the effect of palm biodiesel diffusion on the mechanical response of the rubber remains unexplored.

It is well-known that dry rubber exhibits highly non-linear behavior characterized by large strain and non-linear stress-strain response under static loading. These materials also exhibit a time dependent response which can be demonstrated with creep and relaxation tests. Under cyclic loading, both hysteresis and stress-softening are observed. The hysteresis is given by a different uploading and unloading path and it can be related to viscoelasticity [4] or viscoplasticity [5]. The stress-softening phenomenon or the Mullin's effect [6] occurs exclusively after the first loading of the loading cycles, given by the lower reloading stress. While the mechanical response of the dry rubber is well-documented, the mechanical responses of the swollen rubber are less explored [7].

In this view, the aim of this paper is to study the effect of biodiesel diffusion, which leads to the swelling of rubber, on the mechanical responses of the common rubber components under cyclic loading condition. Two rubber materials are studied: Nitrile Butadiene Rubber (NBR) and Polychloroprene Rubber (CR).

\section{Experimental Program}

Materials. Commercial grade of NBR and CR specimens with 60 shore hardness were fabricated by MAKA Engineering Sdn. Bhd., Malaysia. The specimen is an annular cylindrical block with outside diameter $=50 \mathrm{~mm}$, inner diameter $=38 \mathrm{~mm}$ and height $=10 \mathrm{~mm}$. The palm biodiesel (B100) used in this research was supplied by Am Biofuels Sdn. Bhd., Malaysia. Table 1 shows the analysis report of the palm biodiesel used in the present study. To understand the effect of palm biodiesel diffusion on the mechanical responses of the selected elastomers in detail, a standard Malaysia on-road petroleum diesel fuel (B0) was also tested in this study for comparison. 
Table 1: Properties of B100 palm biodiesel.

\begin{tabular}{lllr}
\hline \hline Test & Unit & Methods & Results \\
\hline \hline Ester content & $\%(\mathrm{~m} / \mathrm{m})$ & EN 14103 & 96.9 \\
Density at $15{ }^{\circ} \mathrm{C}$ & $\mathrm{kg} / \mathrm{m}^{3}$ & EN ISO 12185 & 875.9 \\
Viscosity at $40{ }^{\circ} \mathrm{C}$ & $\mathrm{mm}^{2} / \mathrm{s}$ & EN ISO 3104 & 4.667 \\
Flash point & ${ }^{\circ} \mathrm{C}$ & EN ISO 3679 & 168 \\
Cetane number & - & EN ISO 5165 & 69.7 \\
Water content & $\mathrm{mg} / \mathrm{kg}$ & EN ISO 12937 & 155 \\
Acid value & $\mathrm{mgKOH} / \mathrm{g}$ & EN ISO 3679 & 0.38 \\
Methanol content & $\%(\mathrm{~m} / \mathrm{m})$ & EN 14110 & $<0.01$ \\
Monoglyceride content & $\%(\mathrm{~m} / \mathrm{m})$ & EN 14105 & 0.67 \\
Diglyceride content & $\%(\mathrm{~m} / \mathrm{m})$ & EN 14105 & 0.2 \\
Triglyceride content & $\%(\mathrm{~m} / \mathrm{m})$ & EN 14105 & 0.2 \\
Total glycerine & $\%(\mathrm{~m} / \mathrm{m})$ & EN 14105 & 0.25 \\
\hline \hline
\end{tabular}

Diffusion Test. Stress free diffusion tests for NBR and CR were conducted in diesel (B0) and biodiesel (B100) respectively at room temperature for various diffusion durations: 2, 5, 10, 20 and 30 days. Fig. 1 showed the simple test setup where the specimens were hung and immersed in the stainless steel container. Each specimen was completely immersed in the tested fuel, thereby, allowing stress-free diffusion to occur in the rubber specimens. When the samples reached the desired diffusion duration, they were removed from the container and dipped quickly into acetone to remove the oil excess. The samples were then blotted dry with filter paper before the weight was measured. The percentage of mass change and volume change of the specimens were calculated using the following relations [3]:

$$
\begin{aligned}
& \% \text { Mass change }=\frac{M_{2}-M_{1}}{M_{1}} \times 100 . \\
& \% \text { Volume change }=\frac{\left(M_{2}-M_{4}\right)-\left(M_{1}-M_{3}\right)}{\left(M_{1}-M_{3}\right)} \times 100 .
\end{aligned}
$$

where $M_{1}$ and $M_{2}$ are the mass in air before and after diffusion respectively while $M_{3}$ and $M_{4}$ are the mass in water before and after diffusion respectively.

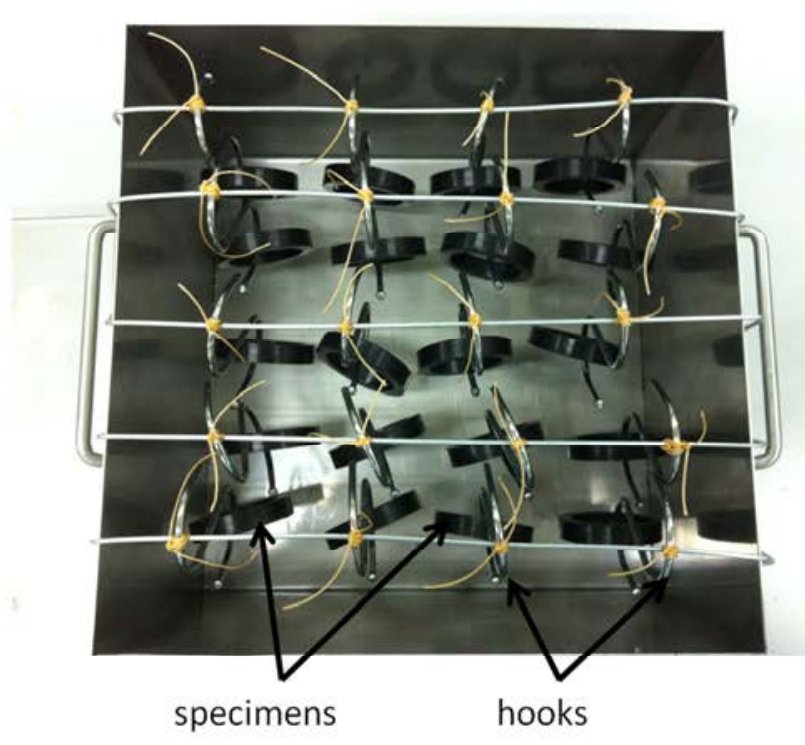

b

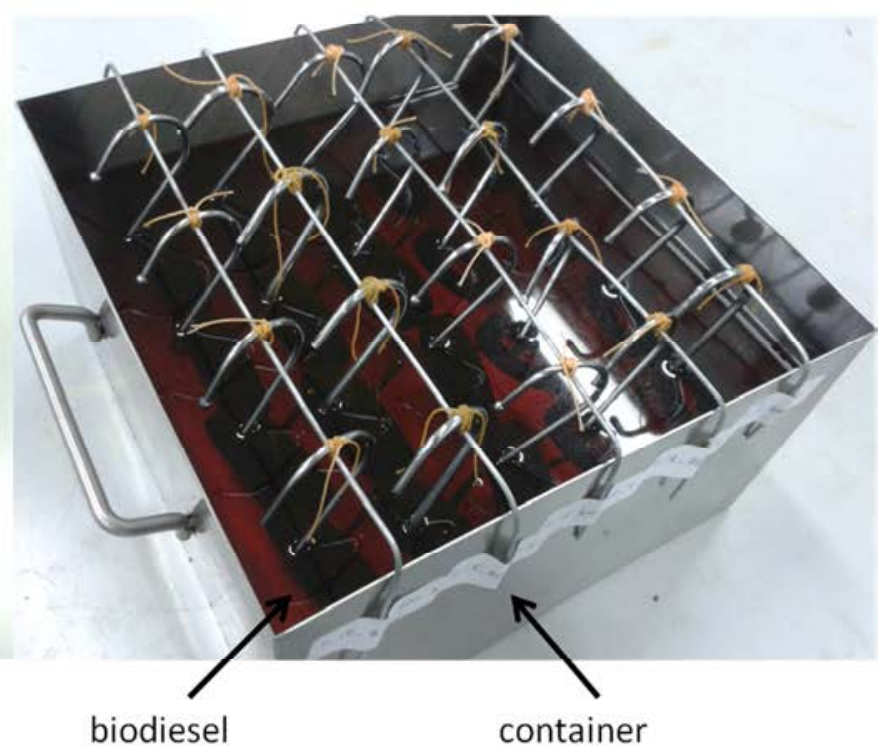

Fig 1: Stress free diffusion test for the specimens (a) before immersion and (b) after immersed in B100. 
Mechanical Test. To gain insight on the effect of biodiesel diffusion, which contributes to the swelling of elastomers, on the mechanical response of elastomers, uniaxial cyclic compressive tests on dry and swollen rubber specimens were carried out using an Instron testing machine operated at room temperature. The experimental setup was connected to a computer to record the experimental data. The tests were conducted at a constant displacement rate of $0.1 \mathrm{~mm} / \mathrm{s}$ to avoid excessive increase in the temperature of the specimens, i.e. the thermal effect is not considered in the present study. Two types of mechanical tests were conducted:

1. Cyclic compressive test. The specimen was subjected to cyclic compressive loading at $40 \%$ maximum compressive strains of 6 cycles each.

2. Multi-relaxation test. After experiencing 6 cycles of compressive loading, the specimen was subjected to relaxation tests at different strain levels during uploading and unloading.

\section{Results and Discussions}

Diffusion. The diffusion of different fuel (B0 and B100) of NBR and CR which is described in terms of mass change and volume change is shown in Fig. 2. Both graphs show similar patterns. The diffusion in both rubber increases when the diffusion time (immersion duration) is increased from 2 days to 30 days. Overall the fuel uptake is relatively fast at the initial stage, especially in biodiesel due to the great affinity in rubber for oil uptake [8]. The rate of swelling, either expressed in terms of mass change or volume change appears to be high at short exposure time before decreasing at longer exposure time. Accelerated diffusion is initiated with absorption of liquids when the liquids dissolve on the surface layer of the rubber (adsorption) until a certain concentration. Subsequently, the liquids penetrate slowly into the rubber by diffusion until the rubber specimen achieves equilibrium swelling [9].

a

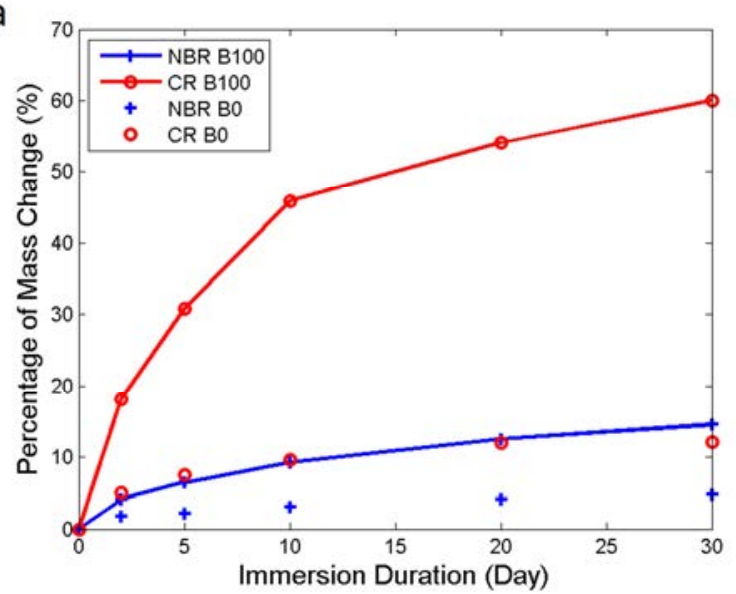

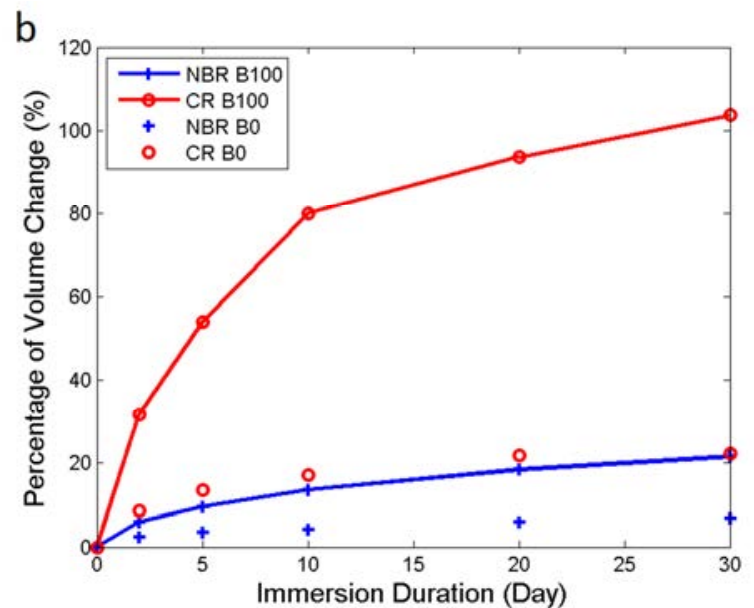

Fig. 2: (a) Mass change and (b) volume change exhibited by NBR and CR after immersion in B100 and $\mathrm{B} 0$ for different immersion durations.

Influence of Diffusion on the Macroscopic Mechanical Response. The stress-strain response of dry and swollen rubbers (after immersed in B0 and B100 for the maximum immersion duration (30 days)) under cyclic compressive loading is presented in Fig. 3. Generally, it is observed that the nature of the stress-strain curves is preserved. However, for a given strain, lower stresses are recorded for swollen rubbers. CR and NBR swollen by B100 showed a lower stress level than the rubbers swollen by B0. Significant decrease of stress in CR after diffused by B100 can be related to the high swelling level due to strong interaction of the rubber-solvent matrix system [10]. Both dry and swollen rubbers exhibit inelastic responses such as stress-softening and hysteresis. The stresssoftening disappears after around five loading cycles while the hysteresis stabilizes after five loading cycles. Moreover, it is to be noted that for both NBR and CR, the inelastic responses appear to be significantly smaller in the case of swollen rubbers. In the next subsections, further discussion on the hysteresis and stress relaxation is presented. 

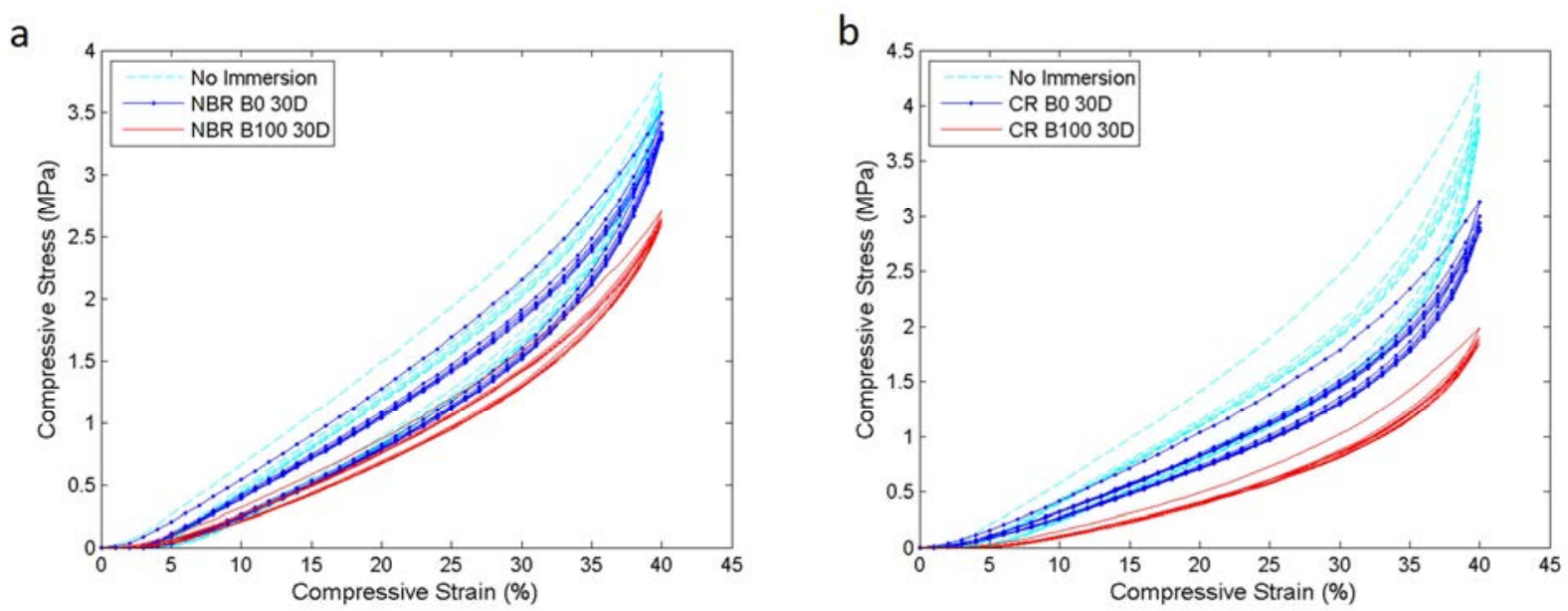

Fig. 3: Stress-strain curves of a) NBR and b) CR at dry (without immersion) and swollen states (after 30 days immersion in B0 and B100). For immersed rubbers, the stress is expressed with respect to the unswollen-unstrained configuration (initial dry cross section).

Hysteresis. The hysteresis in our materials is found to stabilize after five cycles of loading (see Fig. 3 ). In order to investigate the effect of biodiesel diffusion on hysteresis of elastomers, the amount of hysteresis ratio at different immersion durations and different tested fuel is plotted in Fig. 4. Following Bergström and Boyce [4], the hysteresis loss ratio is defined as the amount of hysteresis divided by the supplied energy during uploading. The plot clearly indicates that for both NBR and $\mathrm{CR}$ the hysteresis ratio decreases when the immersion duration increases, i.e. when diffusion increases. The change in hysteresis loss ratio is the least in NBR diffused by B0, only decreased by $16 \%$ after 30 days immersion. Initially, in the dry state CR has the higher hysteresis ratio. As swelling occurs, the hysteresis ratio in CR decreases significantly and becomes consistently lower than that of NBR immersed in the same solvent. These results highlight the decrease of the viscous effect in elastomers as the swelling level increases. Note that in the context of fatigue, Cho et al. [11] attributes the increase of fatigue crack growth in swollen rubbers (decrease in fatigue life) to the decrease of hysteresis.

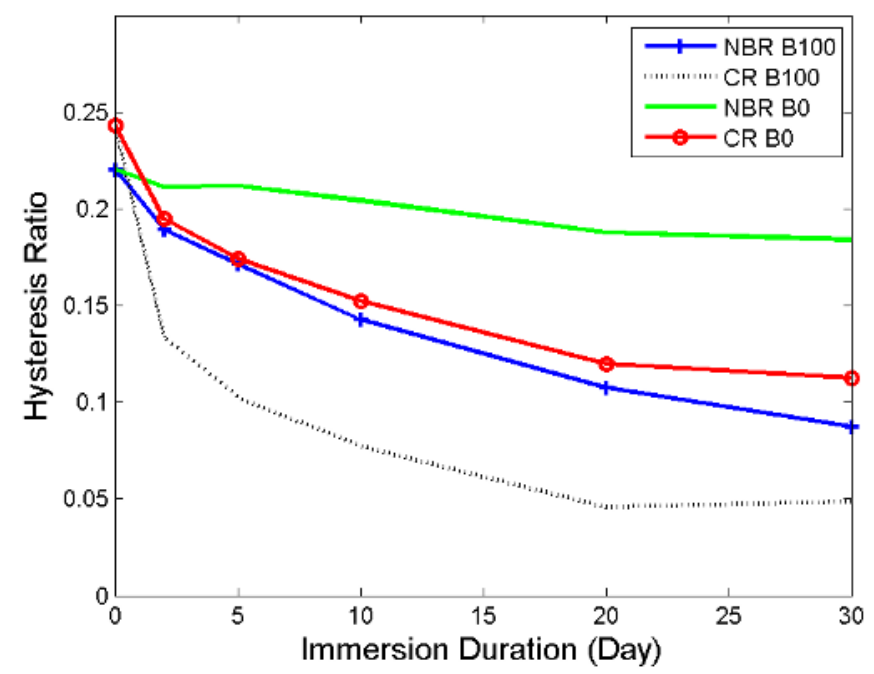

Fig. 4: Hysteresis ratio in NBR and CR under cyclic compressive loading at $40 \%$ maximum strain. Results correspond to 2, 5, 10, 20 and 30 days of immersion duration in B0 and B100 respectively.

Stress-relaxation. The effect of the biodiesel diffusion on the time-dependent behavior of the elastomeric materials can be probed with experiments illustrated in the inset of Fig. 5. In this experiment, after the specimen was pre-conditioned with six cycles of compressive loading to a maximum compressive strain level of $40 \%$, the multi-relaxation tests at strain levels of $10 \%, 20 \%$ and $30 \%$ were conducted during the uploading and unloading. Similar tests were conducted for dry 
elastomers by Lion [5] and Bergström and Boyce [4], among others. It is observed that the compressive stress decreases during the relaxations in the uploading and increases during the relaxations in the unloading approaching what appears to be an equilibrium state [4] as depicted in Fig. 5.

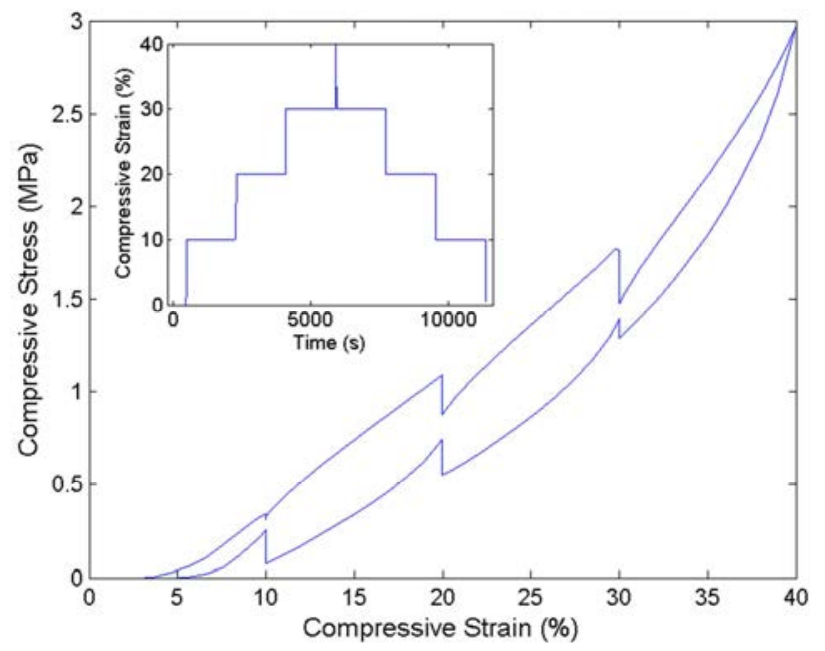

Fig. 5: Engineering stress-strain response of dry NBR under multi step relaxation test at $0.1 \mathrm{~mm} / \mathrm{s}$ displacement rate.

Further investigation on the stress relaxation is presented in Fig. 6 where the normalized stress is plotted as a function of relaxation duration for different immersion durations (diffusion levels). In these figures, the normalized stress is defined by the ratio between the value of stress during relaxation at specific time, $\sigma(t)$ and the maximum stress achieved at the beginning of the corresponding relaxation, $\sigma_{o}$.
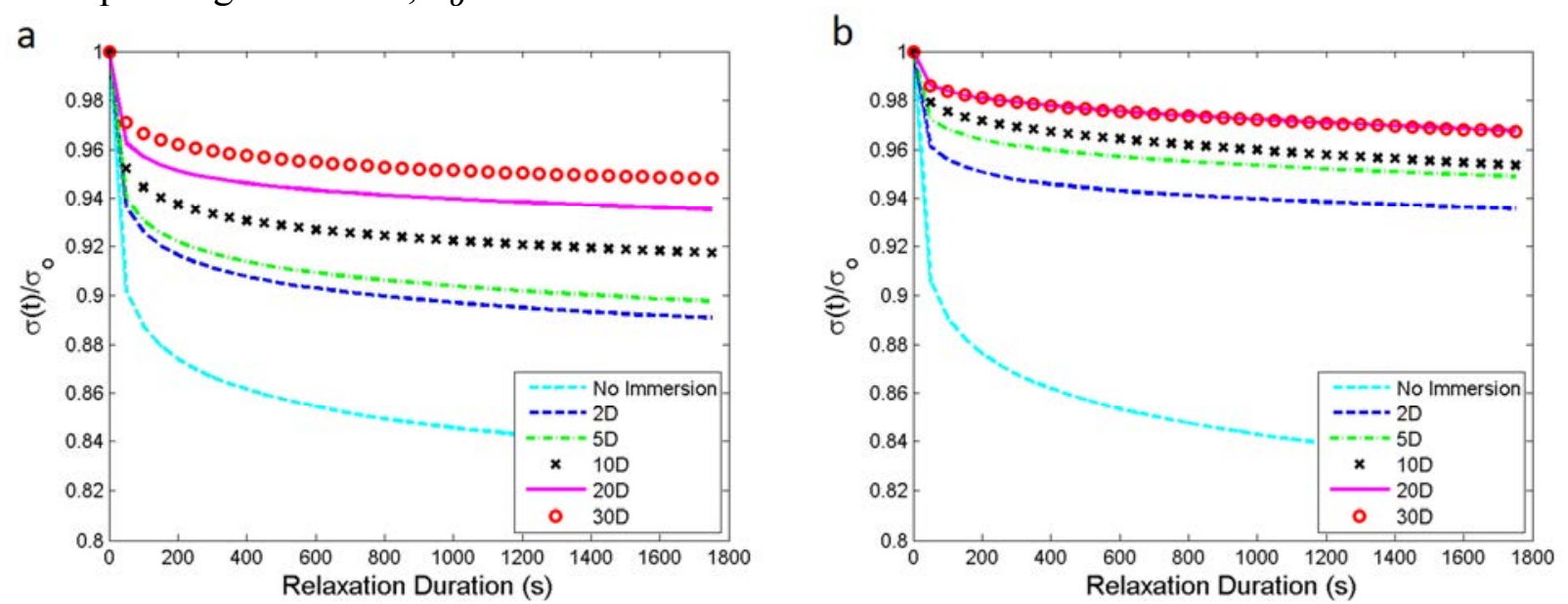

Fig. 6: Stress relaxation of a)NBR and b)CR under compressive strain levels of $30 \%$ during $1800 \mathrm{~s}$. Results correspond to 2, 5, 10, 20 and 30 days of immersion duration in B100.

As clearly shown, the graphs in Fig. 6 show a similar trend. The presence of liquids (diffusion) appears to render the material under loading closer to the equilibrium state, i.e. the viscous (timedependent) contribution to the macroscopic mechanical response of material becomes smaller which is consistent with the results on the hysteresis discussed in the previous subsection. Indeed, after $1800 \mathrm{~s}$ of relaxation, the stress decreases by the amount of $16 \%$ for dry NBR and by $6 \%$ whenever the specimen is immersed in B100 for 30 days. While for CR, the stress only decreased by $3 \%$ after immersed for 30 days in B100 whereas the stress in dry CR decreased by $17 \%$ after $1800 \mathrm{~s}$ of relaxation. As emphasized by Radhakrishnan Nair [12]; Wypych [13], the palm biodiesel appears to act as a lubricant facilitating movement of the polymer chain segments. 


\section{Summary}

In the present work, a simple experimental setup was developed to probe the mechanical responses of swollen rubbers under cyclic compressive loading conditions. Different levels of swelling due to biodiesel diffusion resulting from different immersion durations of elastomeric specimens were considered. It was found that the diffusion rate is relatively high at short immersion durations and becomes lower at longer immersion duration. The diffusion in CR was systematically higher than the one in NBR for the given immersion duration.

The diffusion of biodiesel in the rubber appeared to reduce the strength of rubber. Under cyclic loading conditions, both swollen NBR and swollen CR exhibited inelastic responses, i.e. stresssoftening, hysteresis and stress relaxation. However, the amounts of these inelastic responses in biodiesel diffused rubbers were significantly lower than that found in dry rubbers.

To close the paper, it is to be noted that the present works focuses only on stress-free diffusion. Further investigations on the effect of multiaxial stress state on the diffusion and the resulting mechanical response are needed.

\section{Acknowledgement}

The authors greatly appreciate the financial support from Ministry of Higher Education Malaysia through High Impact Research Grant HIR-MOHE D000008-16001.

\section{References}

[1] A.S.M.A. Haseeb, H.H. Masjuki, C.T. Siang, M.A. Fazal: Renew. Energ. Vol. 35 (2010), p. 2356-2561.

[2] A.S.M.A. Haseeb, T.S. Jun, M.A. Fazal, H.H. Masjuki: Energy Vol. 36 (2011), p. 1814-1819.

[3] W. Trakarnpruk, S. Porntangjitlikit: Renew. Energ. Vol. 33 (2008), p. 1558-1563.

[4] J. S. Bergström, M. C. Boyce: J. Mech. Phys. Solids Vol. 46 (1998), p. 931-954.

[5] A. Lion: J. Mech. Phys. Solids Vol. 45 (1997), p. 1805-1834.

[6] L. Mullins: Rubber Chem. Technol. Vol. 21 ( 1948), p. 281-300.

[7] A.B. Chai, A. Andriyana, E. Verron, M.R. Johan, A.S.M.A. Haseeb: Polym. Test Vol. 30 (2011), p. 867-875.

[8] A. Mostafa, A. Abouel-Kasem, M.R. Bayoumi, M.G. El-Sebaie: Mater. Design Vol. 30 (2009), p. 1561-1568.

[9] A.N. Gent: Engineering with rubber. How to design rubber components (Hanser Publishers, Germany 2001).

[10] S.C. George, S. Thomas: Prog. Polym. Sci. Vol. 26 (2001), p. 985-1017.

[11] K. Cho, W.J. Jang, D. Lee, H. Chun, Y.-W. Chang: Polymer Vol. 41 (2000), p. 179-183.

[12] N. Radhakrishnan Nair. Studies on depolymerized natural rubber. PhD thesis: Mahatma Gandhi University (1997).

[13] G. Wypych. Handbook of plassticizers (ChemTec Publishing, 2004). 